\title{
Nanobodies raised against monomeric a-synuclein inhibit fibril formation and destabilize toxic oligomeric species
}

\author{
Marija Iljina ${ }^{1 \dagger}$, Liu Hong ${ }^{1,2}$, Mathew H. Horrocks ${ }^{1,3}$, Marthe H. Ludtmann ${ }^{4}$, Minee L. Choi ${ }^{4}$, Craig D. Hughes ${ }^{5}$, \\ Francesco S. Ruggeri ${ }^{1}$, Tim Guilliams ${ }^{1,6}$, Alexander K. Buell ${ }^{1,8}$, Ji-Eun Lee', Sonia Gandhi ${ }^{1}$, Steven F. Lee ${ }^{1}$, \\ Clare E. Bryant ${ }^{5}$, Michele Vendruscolo ${ }^{1}$, Tuomas P. J. Knowles ${ }^{1}$, Christopher M. Dobson ${ }^{1 *}$, Erwin De Genst ${ }^{1,7^{*}+}$ \\ and David Klenerman ${ }^{1 *}$
}

\begin{abstract}
Background: The aggregation of the protein a-synuclein (aS) underlies a range of increasingly common neurodegenerative disorders including Parkinson's disease. One widely explored therapeutic strategy for these conditions is the use of antibodies to target aggregated aS, although a detailed molecular-level mechanism of the action of such species remains elusive. Here, we characterize as aggregation in vitro in the presence of two aS-specific single-domain antibodies (nanobodies), NbSyn2 and NbSyn87, which bind to the highly accessible C-terminal region of as.

Results: We show that both nanobodies inhibit the formation of aS fibrils. Furthermore, using single-molecule fluorescence techniques, we demonstrate that nanobody binding promotes a rapid conformational conversion from more stable oligomers to less stable oligomers of as, leading to a dramatic reduction in oligomer-induced cellular toxicity.
\end{abstract}

Conclusions: The results indicate a novel mechanism by which diseases associated with protein aggregation can be inhibited, and suggest that NbSyn2 and NbSyn87 could have significant therapeutic potential.

Keywords: Protein aggregation, Amyloid toxicity, Neurodegeneration, Aggregation inhibitors, Antibody, Singlemolecule fluorescence

\section{Background}

The aberrant aggregation of the protein a-synuclein $(\mathrm{aS})$ has been linked to the onset and progression of Parkinson's disease (PD), dementia with Lewy bodies [1], PD dementia, multiple system atrophy, and related synucleopathies [2-4]. The histopathological characteristics of PD and its associated disorders include the presence of neuronal inclusions, for example, Lewy bodies and Lewy neurites, which are primarily composed of fibrillar aS $[1,5]$. The deposition of aS in the nervous system follows a characteristic pattern [6], and the ability of

\footnotetext{
*Correspondence: cmd44@cam.ac.uk; erwin.degenst@astrazeneca.com; dk10012@cam.ac.uk

${ }^{\dagger}$ Equal contributors

'Department of Chemistry, University of Cambridge, Lensfield Road,

Cambridge CB2 1EW, UK

Full list of author information is available at the end of the article
}

aggregated species to propagate across the brain by a mechanism that is defined as prion-like is increasingly recognized [7]. In aqueous solution, monomeric aS self-assembles into amyloid fibrils resembling those that are deposited in the brain, and the aggregation process proceeds through the formation of intermediate species, including soluble oligomers, prior to fibril formation $[8,9]$. Moreover, these oligomeric species, rather than mature amyloid fibrils, have increasingly been identified as the most highly neurotoxic forms of aS [10-16].

As a consequence of the central role of aS aggregation in PD and the neurotoxicity of its assemblies, immunotherapy against $\mathrm{aS}$ is being widely pursued as a potential disease-modifying strategy $[17,18]$. Although passive immunization using antibodies targeting aS has shown promise in numerous in vitro and in vivo model systems, 
as well as in several clinical trials $[19,20]$, there is no effective treatment for PD and other synucleinopathies. In order to make significant advances in this objective, it is crucial to develop a detailed understanding of the molecular mechanism of action of aS-specific antibodies during the aggregation process of the protein.

Over the years, full-length antibodies and antibody fragments, such as scFvs, have been generated against different regions and different species of aS for either biophysical studies, target validation, or therapy (reviewed in De Genst et al. [21] and Bergstrom et al. [20]), and have been used in multiple reported studies [22-27].

Full-length aS consists of 140 amino acid residues divided into three distinct regions, namely a positively charged lipid-binding $\mathrm{N}$-terminal segment comprising residues $1-60$, a central hydrophobic segment consisting of residues 61-95, and a negatively-charged C-terminal region comprising residues 96-140 [28]. This latter region does not include the residues observed to be in the amyloid fibril core [29], although previous in vitro studies have shown that its deletion promotes aS aggregation [30], indicating its involvement in the aggregation process. Notably, antibodies directed to the C-terminal region of aS, or specific for oligomeric forms of aS, were reported both to suppress strongly its aggregation and to reduce cellular toxicity $[24,27]$.

We have previously described two single-domain fragments of camelid heavy-chain antibodies [31], termed 'nanobodies' [32], denoted NbSyn2 [33] and NbSyn87 [34], which bind to distinctive epitopes within the C-terminal region of monomeric aS (residues 137-140 and 118-131, respectively). These nanobodies, which were raised against and bind to monomeric aS, also recognize fibrillar forms of $a S$ in which the C-terminal region is exposed [29], allowing the use of these nanobodies as biophysical probes to study the properties of aS fibrils [34]. Moreover, one of these nanobodies, NbSyn87, when expressed as a genetic fusion protein with a PEST proteasomal degradation tag, was found to reduce toxicity in cell-lines overexpressing aS as a result of the specific degradation of monomeric aS, thereby significantly reducing proteostatic stress [35].

In order to understand such phenomena in molecular detail, it is important to characterize the impact of nanobody binding on the overall aS aggregation process in vitro, and in particular its effect on the formation of the highly toxic oligomers that are linked to cellular damage. It is known from previous studies that the C-terminal region of aS is exposed in at least some of its aggregated intermediate forms, such as oligomers [36], as well as monomers and fibrils, and that targeting of this region by antibodies has been recognized to have protective effects [19]. Therefore, it is plausible that the nanobodies and other C-terminal-binding antibodies can affect the formation and properties of these oligomers.

Since the pre-fibrillar oligomers are generally transient and heterogeneous, and are typically present at very low concentrations relative to aS monomers, they are difficult to study by conventional bulk techniques. We have previously utilized single-molecule Förster resonance energy transfer (sm-FRET) measurements in order to characterize the oligomerization of aS in considerable detail $[13,37]$. Using this technique, we identified two distinct types of aS oligomers that were populated sequentially prior to the formation of aS fibrils [13]. The two oligomer types had distinct FRET efficiency distributions and were therefore termed "low-FRET" and "high-FRET" oligomers [13]. The differences in their photophysical properties, as well as their different kinetics of formation, stabilities towards dissociation and enzymatic proteolysis, and toxicity to cells indicated that these two oligomer forms were structurally distinct intermediates generated in the process of the formation of aS fibrils [13, 38]. In particular, the initial assembly of low-FRET oligomers was followed by slow conformational conversion into high-FRET oligomers prior to fibril formation. The accumulation of high-FRET oligomers was associated with the highest level of cytotoxicity, pointing to this specific oligomer type as the most damaging species formed during aS aggregation.

In this study, we set out to characterize in detail the effects of NbSyn2 and NbSyn87 on the aggregation of aS in vitro. First, we investigated fibril formation using a combination of biophysical experiments to monitor the kinetics of this process, and compared the properties of the various resulting fibrillar aggregates. Second, we employed sm-FRET techniques to explore how the presence of NbSyn87 and NbSyn2 affects the formation of the low-FRET and high-FRET oligomers. We identified the ability of the nanobodies to inhibit the formation of aS fibrils and to destabilize toxic high-FRET oligomers of aS, and explored how the latter process affects oligomer-induced cytotoxicity.

\section{Results}

NbSyn2 and NbSyn87 inhibit the formation, maturation, and elongation of aS fibrils

We first investigated the effects of the aS-specific nanobodies, NbSyn 87 and NbSyn2, on aS fibril formation. To determine whether or not the binding of nanobodies to the $\mathrm{C}$-terminal region affects the aggregation propensity of aS, we initially performed bulk solution aggregation assays to monitor fibril formation by full-length wildtype (wt) aS using thioflavin $\mathrm{T}$ (ThT) fluorescence measurements to monitor the quantity of aggregated aS. In these experiments, $\mathrm{aS}$ at a concentration of $70 \mu \mathrm{M}$ was aggregated in the absence or presence of $140 \mu \mathrm{M}$ 
NbSyn2, NbSyn87, or a control nanobody, cAbHuL5g (NbHul5g) [39]; the latter is a lysozyme-specific nanobody [39] and is therefore not expected to bind to aS.

From Eq. 1 (Methods), taking the dissociation constants of NbSyn2 and NbSyn87 for monomeric aS, respectively, as approximately $264 \mathrm{nM}$ and $42 \mathrm{nM}$ [34] at $37^{\circ} \mathrm{C}$, we calculated that the free aS concentration under these conditions was approximately $260 \mathrm{nM}$ and $40 \mathrm{nM}$, respectively, thereby approximating full saturation of all aS binding sites. The samples were incubated at physiologically relevant $\mathrm{pH}$ and ionic strengths (as detailed in the Methods section) at $37{ }^{\circ} \mathrm{C}$ with agitation to promote fibril formation; the resulting kinetic profiles of fibril formation are shown in Fig. 1a.

The aggregation kinetics of aS in the absence of nanobodies are in good agreement with our previously reported bulk kinetic measurements of aS under similar incubation conditions [13, 37]. In contrast, moderate inhibition of fibril formation was observed in the presence of NbSyn2, whereas solutions incubated in the presence of NbSyn 87 gave rise to much lower ThT fluorescence signals over the entire duration of the experiment, indicating a stronger inhibition of aS fibril formation in the presence of this nanobody [13, 37]. In addition, we confirmed the inhibition of the formation of ThT-active aggregates in the presence of NbSyn 2 and NbSyn 87 by total internal reflection fluorescence microscopy imaging of the solutions extracted during the process of the aggregation reaction (Additional file 1: Figure S1).

Subsequently, we characterized the end products of aggregation using atomic force microscopy (AFM). In agreement with the findings from the ThT fluorescence assays, the images obtained in this way showed the presence of large numbers of amyloid fibrils in the solutions of aS that had been incubated in the absence of nanobodies or in the presence of NbHul5g or NbSyn2 (Fig. 1b). In the samples of aS incubated with NbSyn87, only small and apparently spherical aggregates along with monomeric species were observed, and no large fibrils could be detected (Fig. 1b). Comparison of average fibril heights from AFM maps revealed that fibrils formed in the presence of NbSyn2 were thinner than fibrils formed in the absence of nanobodies or in the presence of NbHul5g (Fig. 1c and Additional file 1: Figure S1), indicating that nanobodies impaired the maturation of aS fibrils. The differences in the fibril-forming capability of aS identified in the presence of the two aS-specific nanobodies might be due to the differences in the epitopes they bind. NbSyn 87 binds with higher affinity and closer to the NAC region than does NbSyn2, and thereby might slow fibril formation due to steric hindrance and promote the formation of smaller fibrils (Fig. 1b).
We also performed quartz crystal microbalance experiments $[40,41]$ in order to measure the effects of NbSyn2 and NbSyn87 on the elongation rates of pre-formed aS fibrils upon their incubation with either monomeric aS, or with a stoichiometric ratio of monomeric aS and NbSyn87, NbSyn2, or NbHul5g (Additional file 2). It was found that the elongation rate of aS fibrils markedly decreased in the presence of both aS-specific nanobodies, corresponding to $30-40 \%$ of the rate in their absence, as described in further detail in Additional file 2: Tables S3 and S4, and discussed in Additional file 2: Supplementary Results.

\section{NbSyn2 and NbSyn87 impede the generation of high-FRET oligomeric species of aS prior to fibril formation}

Having characterized the impact of the aS-specific nanobodies on the formation of aS fibrils, we subsequently set out to focus on the earlier steps in the aS aggregation reaction and to determine whether or not nanobodies affected oligomer formation. To this end, we used smFRET techniques developed in previous studies of the aggregation of $\mathrm{aS}$ in aqueous solution [13, 37, 38]. In sm-FRET experiments, an alanine to cysteine mutation at residue 90 of aS (A90C) was introduced to enable the attachment of a single Alexa Fluor (AF) dye; we have previously shown that this modification does not significantly influence the kinetics of aS fibril formation $[13,37,38]$, a conclusion additionally confirmed in the present study by bulk ThT assays using wt and AF-labeled aS (Additional file 3: Figure S2a). Because residue 90 is located at the periphery of the central amyloidogenic region of the aS molecule [28], AF dyes come into close proximity during fibril formation. We have used AF488 and AF594 dyes, which we have found are sufficiently close together within the aggregates for FRET to be detectable, the efficiency of which is a useful readout of the aggregation reaction (Eq. 2, Methods). This approach makes it possible to detect two distinct oligomer types, those with a low FRET efficiency and those giving rise to a higher FRET efficiency, both of which are observed prior to fibril formation [13, 37, 38]. The smFRET analysis is selective for oligomeric aggregates and excludes large fibrillar species formed later during the aggregation process (as detailed in Methods and demonstrated in previous studies [13, 37, 38]). Therefore, this technique is complementary to the bulk ThT assay that has been used to monitor fibril formation (Fig. 1a). For every oligomer detected in the experiment, the FRET efficiency value and the apparent size in monomer units were calculated according to Eqs. 2 and 3 (Methods). These data are represented in Fig. 2 as histograms to show both the numbers and properties of the oligomer distributions. 


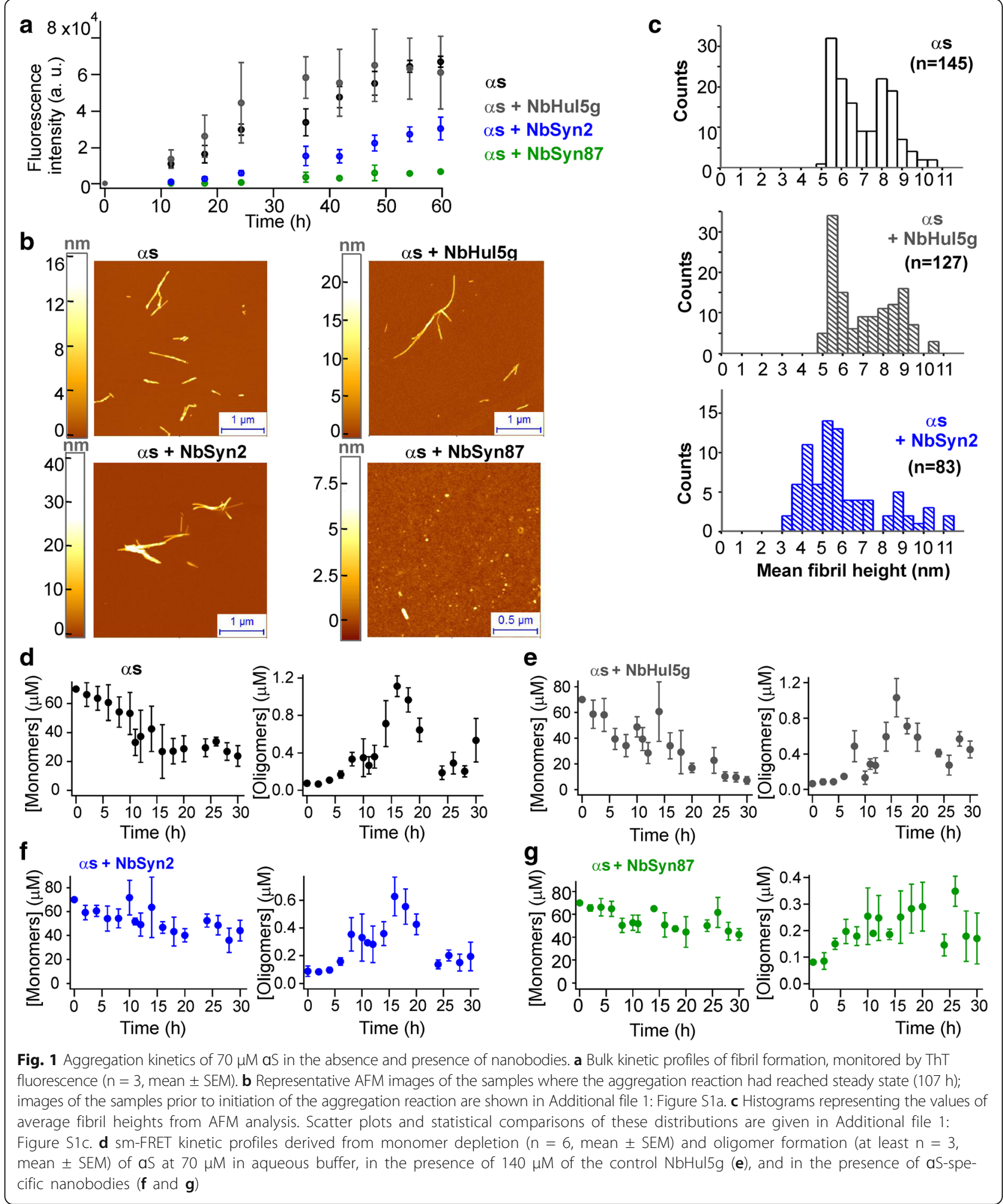

In sm-FRET experiments, equimolar mixtures of AF488labeled aS (AF488-aS) and AF594-labeled aS (AF594-aS) were used at a total protein concentration of $70 \mu \mathrm{M}$, either in the presence or absence of $140 \mu \mathrm{M}$ of unlabeled nanobodies. The solutions were incubated with agitation under the same conditions as in the bulk ThT assays, and aliquots were withdrawn at regular time intervals during the incubation, diluted $10^{5}$-fold to generate appropriate 


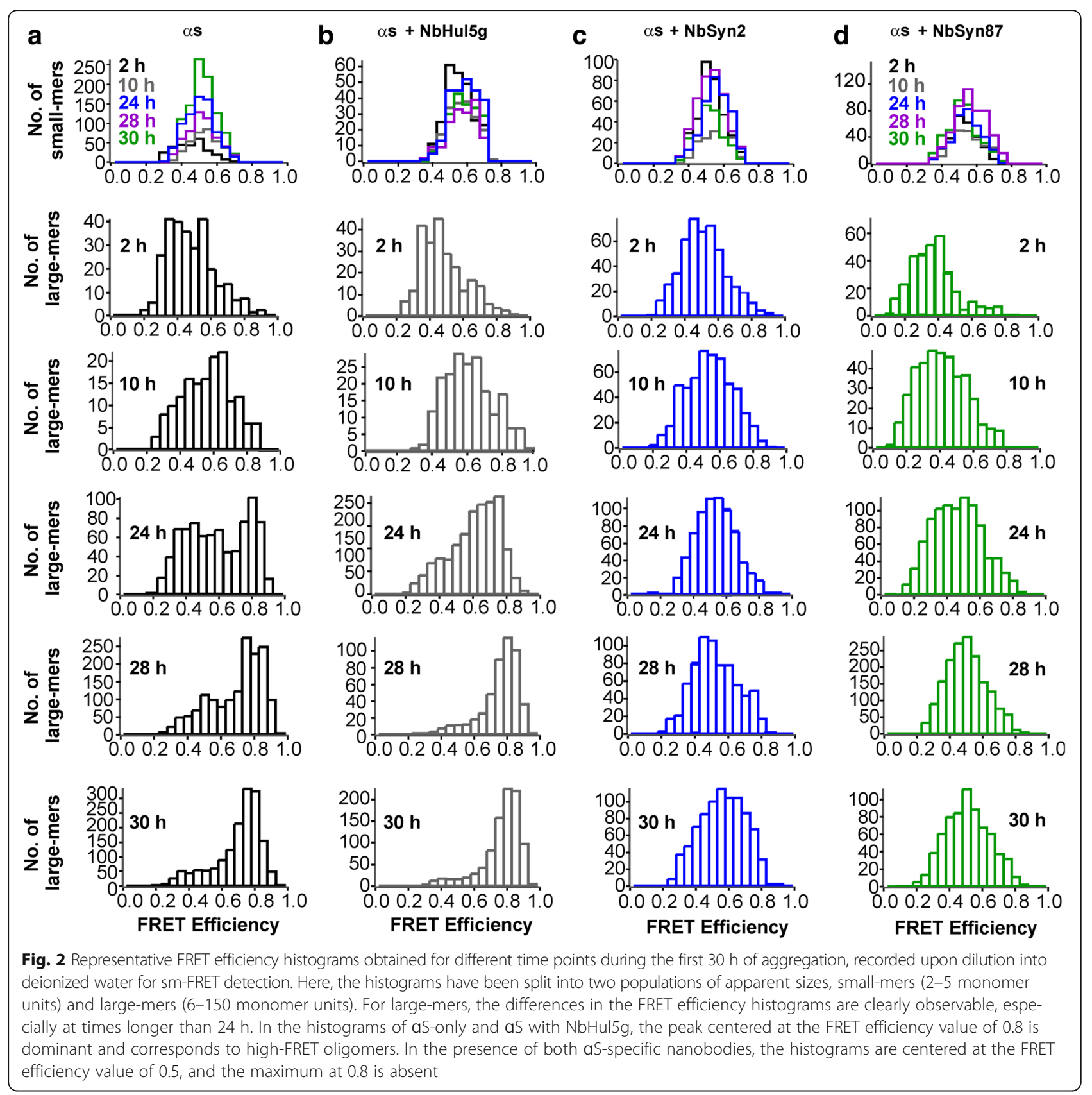

conditions for single-molecule detection, and immediately analyzed by sm-FRET. We analyzed the solutions both following their dilution into aqueous buffer of the same composition as that used for the incubations (PBS, see in Methods), and following dilution into deionized water; we have recently shown that dilution into low ionic strength solutions enables an improved separation of the low- and high-FRET oligomers [38]. We measured the average brightness of AF488-aS and AF594-aS in the presence of the unlabeled nanobodies to verify that the nanobodies did not affect the fluorescence properties of the dyes in the sm-FRET experiments (Additional file 2: Supplementary
Information). In addition, we carried out control experiments using samples where both aS and nanobodies were labeled with AF to confirm that the nanobodies were fully dissociated from aS during the detection step under both solution conditions; the results were consistent with previously reported $K_{d}$ values [34], as detailed in Additional file 2: Supplementary Methods and Tables S1 and S2.

The resulting kinetic profiles of $\mathrm{aS}$ monomer depletion and oligomer formation are shown in Fig. 1d-g. The most rapid decrease in the monomer concentration, deduced from the monomer burst-rates [37], occurred in the samples containing $70 \mu \mathrm{M}$ aS alone or in the 
presence of the control NbHul5g (Fig. 1d, e). However, samples containing NbSyn87 and NbSyn2 showed significantly slower monomer depletion. Based on the monomer depletion data derived from sm-FRET, after $30 \mathrm{~h}$ of aggregation, approximately $70 \%$ of the sample was aggregated in the aS-only reaction, $90 \%$ in the aS plus control nanobody, and approximately $40 \%$ in the samples containing both aS and NbSyn 87 or NbSyn2. The oligomers were formed in all samples, and comprised less than $2 \%$ of the samples of aS (Fig. 1d-g).

Transmission electron microscopy (TEM) images of the samples at incubation times longer than $100 \mathrm{~h}$ confirmed the presence of abundant quantities of amyloid fibrils in the solutions containing aS alone or in the presence of NbHul5g and of NbSyn2, while only oligomers and short protofibrils were detected in the presence of NbSyn87 (Additional file 3: Figure S2b). These results confirm that the nanobodies inhibit aS fibril formation, and are therefore in good agreement with the bulk ThT fluorescence data and AFM results obtained for wt unlabeled aS as detailed above (Fig. 1b).

To elucidate the effects of the nanobodies on the formation of the low-FRET and high-FRET oligomers, individual FRET efficiency histograms of the samples recorded following dilution into water were analyzed. These showed clear differences between the control samples and the samples containing aS-specific NbSyn2 and NbSyn87 (Fig. 2). As in our previous work [13, 37], we separated the FRET efficiency histograms into two groups, corresponding to the histograms derived from "small" oligomers containing 2-5 apparent monomer units (denoted as "small-mers") and oligomers containing 6-150 apparent monomer units ("large-mers"), based on the number of peaks that were resolvable in the FRET efficiency histograms, as previously reported [37]. This procedure allows us to identify the time-dependent changes readily, which are most clearly evident in the histograms for the large oligomers formed during aggregation (Fig. 2).

For the samples of aS alone, and $\mathrm{aS}$ in the presence of control NbHul5g, two peaks could be observed in the FRET efficiency histograms of the large-mers (Fig. 2), corresponding to the two previously identified oligomer populations [13, 37, 38]. Low-FRET oligomers are characterized by a population centered at an average FRET efficiency value (E) of 0.5 , and high-FRET oligomers are indicated by the peak centered at $\mathrm{E}$ value of approximately 0.8 (Fig. 2). The high-FRET population was clearly distinguishable after $24 \mathrm{~h}$ of aggregation and was dominant by $30 \mathrm{~h}$ of incubation both in the absence of nanobodies and in the presence of the control nanobody (Fig. 2), in good agreement with our previously reported kinetics of high-FRET oligomer formation [13, 37]. The mild acceleration of the formation of high-FRET species in the presence of the control nanobody NbHul5g compared to the aS-only sample might be due to the crowding effect or transient interactions of NbHul5g with aS. Instead of exhibiting two populations at times longer than $24 \mathrm{~h}$, however, the FRET histograms of oligomers formed in the presence of aS-specific nanobodies showed a single broad peak. The appearance of these histograms resembles those that had been obtained previously for aS solutions at low concentrations, in which high-FRET oligomers were not formed at high levels, yet were not fully absent [37]. Therefore, the appearance of the FRET efficiency histograms suggests that, in the presence of aS-specific nanobodies, the formation of high-FRET oligomers is impeded.

To characterize quantitatively the observed differences in aS oligomer populations either in the absence or presence of the nanobodies, we carried out a detailed kinetic analysis by fitting the experimental aggregation profiles to a nucleation-conversion-polymerization model similar to that reported previously [37]. A description of the analysis and the rate constants obtained for the individual microscopic steps of the aggregation process are available in Additional file 2: Supplementary Information and Additional file 4: Figure S3. We achieved the closest agreement with experimental data by assuming that both of the aS-specific nanobodies accelerate the reverse microscopic reaction steps, i.e., the conformational conversion from high-FRET oligomers to low-FRET oligomers and subsequently to monomers. This increase in rate results in the net inhibition of the overall forward aggregation process in the presence of NbSyn2 and NbSyn87, and successfully explains the experimentally observed reduced proportion of high-FRET oligomers and the altered kinetics of monomer depletion and fibril formation. Furthermore, this analysis predicted the distinct accumulation of low-FRET oligomers over longer aggregation times (Additional file 4: Figure S3). In the following section we discuss direct evidence for these phenomena.

\section{NbSyn2 and NbSyn87 enhance the conversion of high-FRET into low-FRET oligomeric species}

Having characterized the kinetics of aS aggregation in the presence of NbSyn2 and NbSyn87, we set out to obtain direct evidence to support the conclusion that the nanobodies could promote the conformational conversion step from high-FRET to low-FRET species. To this end, we tested whether or not the addition of the nanobodies promoted the dissociation of pre-formed high-FRET oligomeric species. For these experiments, solutions from the aggregation reactions of $70 \mu \mathrm{M}$ labeled aS in the absence of nanobodies were collected after $29 \mathrm{~h}$ of incubation and analyzed by sm-FRET to monitor the presence of high-FRET oligomers. 
Subsequently, two molar equivalents of unlabeled NbSyn2, NbSyn 87 or NbHul5g were added to these samples prior to sm-FRET measurement. The representative contour plots of FRET efficiencies and apparent oligomer sizes, as well as the FRET efficiency histograms of each sample (Fig. 3) show highly reproducible results as nearly identical outcomes were observed in at least five independent experiments where fresh nanobodies were added to pre-formed high-FRET oligomers. The addition of NbSyn2 or NbSyn87 resulted in a reproducible decrease of the mean FRET efficiency values (Fig. 3b). These changes in FRET efficiencies cannot be explained by optical effects, e.g., quenching of fluorescence due to the binding of the nanobodies to the oligomeric species, because the samples were diluted by a factor of $10^{5}$, bringing the concentration of the NbSyn87 and NbSyn2 significantly below the Kd values of the interaction of the nanobodies with monomeric aS, as in all previous sm-FRET measurements [34]. In addition, the $k_{\text {off }}$ values for NbSyn87 and NbSyn2 are the order of 0.01 and $0.1 \mathrm{~s}^{-1}$, respectively (Additional file 2 : Supplementary Methods), indicating that the complexes would dissociate upon dilution, an assumption that was confirmed by the experimentally observed absence of coincidence between the labeled nanobody and aS under our detection conditions.

The changes in FRET efficiency values following the addition of NbSyn2 or NbSyn87 can therefore be attributed solely to the nanobody-induced conformational conversion of the high-FRET oligomers to the lessordered low-FRET oligomers, in agreement with the results of the kinetic analysis. This conversion process was particularly fast in the presence of NbSyn87, with a rate constant of $1.0 \pm 0.5 \mathrm{~h}^{-1}$ (Additional file 4: Figure S3); the more highly pronounced effect of NbSyn87 compared to NbSyn2 in this experiment is attributable to its higher affinity for aS. The oligomers will remain in the low-FRET structure during the analysis as the conformational reorganization from low-FRET to high-FRET oligomers in the absence of the nanobodies is characterized by a high energy barrier, reflected in a half-time of several hours [13].

The conformational conversion from high- to lowFRET oligomers described here was observed upon the addition of two molar equivalents of nanobodies. We also carried out titrations to determine the lowest stoichiometric ratio of the nanobodies to $\mathrm{aS}$ at which this behavior is observable. The reduction in FRET efficiencies in this experiment could be clearly observed down

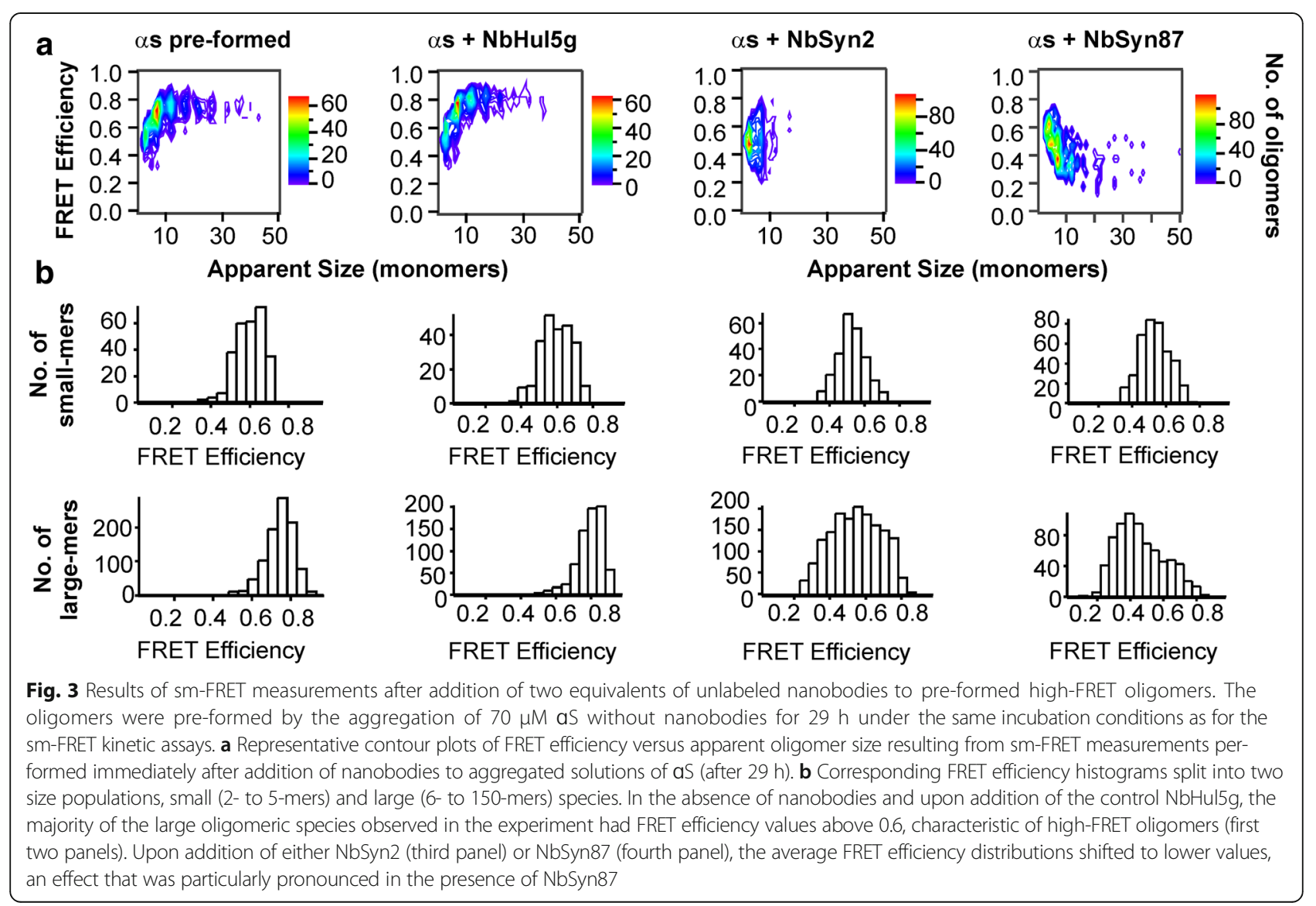


to 0.5 molar equivalents of the nanobodies, while at concentrations below 0.25 molar equivalents this process was too slow to be observed even upon incubation for $10 \mathrm{~h}$ (Additional file 5: Figure S4).

The reduced population of high-FRET oligomers in the presence of NbSyn87 and NbSyn2 leads to significantly reduced cellular damage

The observation that NbSyn 2 and NbSyn87 impair the formation of high-FRET oligomer types during the aggregation process and induce a more rapid conformational conversion of pre-formed high-FRET oligomers into low-FRET species, prompted us to test if these effects could result in an overall decrease in the average stability and cytotoxicity of the oligomers generated at the early stages of the aggregation process of aS.

It has previously been shown that high-FRET oligomers are more resistant towards digestion by proteinase
$\mathrm{K}$ than are low-FRET oligomers, an observation that serves as an additional indication of the structural differences between these two oligomer types [13]. To determine whether or not the populations of oligomers formed in the presence of the nanobodies were more susceptible to proteinase $\mathrm{K}$ digestion than these formed in their absence, we exposed aS samples after $29 \mathrm{~h}$ of incubation under aggregating conditions to varying concentrations of proteinase $\mathrm{K}$. The resulting digestion profiles are shown in Fig. 4a, and representative contour plots of FRET efficiencies and size distributions derived for individual samples in this assay are given in Additional file 6: Figure S5. From Fig. 4a, the populations of oligomeric species formed in the presence of NbSyn 2 or NbSyn 87 were more susceptible to proteinase $K$ digestion than those formed in the presence of $\mathrm{NbHul5g}$. This result is consistent with a lower proportion of more compact high-FRET oligomers in the solutions



Fig. 4 Comparative assays showing the relative stability and cytotoxicity of aS oligomers generated after aggregation for $29 \mathrm{~h}$ in the presence or absence of nanobodies under the same conditions as for the sm-FRET measurements. a Sm-FRET proteinase K digestion assays. The fraction of degradation is the number of oligomers in the proteinase K-exposed sample divided by the number of oligomers in the initial sample $(n=3$, mean \pm SD). Additional file 6: Figure S5 shows representative contour plots of FRET efficiencies versus apparent oligomer sizes. $\mathbf{b}$ Degradation of the $29 \mathrm{~h}$ samples by the 265 proteasome over $24 \mathrm{~h}(\mathrm{n}=3, \mathrm{SD}, P<0.05)$, analyzed by sm-FRET. c Cytoplasmic reactive oxygen species (ROS) production measured by monitoring the rate of dihydroethidium fluorescence (detailed in Additional file 2: Supplementary Methods). Application of aS oligomers to mixed cultures of rat hippocampal and cortical neurons (500 nM of total aS) induced an increase in the rate of ROS production relative to a control with no aS (taken as 100\%); aS alone ( $\mathrm{n}=76$ cells); aS with NbHul5g ( $\mathrm{n}=66$ cells); aS with NbSyn2 $(\mathrm{n}=89$ cells, $P<$ 0.001); and aS with NbSyn87 ( $n=54$ cells, $P<0.01$ ). Additional file 8: Figure 56 shows representative plots of dihydroethidium fluorescence versus time. $\mathbf{d}$ Percentage cell death, measured by propidium iodide staining after incubation with the different aS oligomeric species. Cell death in the absence of aS (buffer control, $n=6$ coverslips); upon incubation with aS alone (100 nM total aS, $n=7$ coverslips); with aS with NbSyn2 ( $n=5$ coverslips, $P<0.001$ ); with aS with NbSyn87 ( $n=8$ coverslips, $P<0.05$ ). e Quantification of the released pro-inflammatory cytokine TNF-a in BV2 microglia after the application of $29 \mathrm{~h}$ timepoints of aS (10 $\mu \mathrm{M}$ of the total aS), aggregated in the absence of nanobodies $(\mathrm{n}=3$, SEM), in the presence of $2 \mathrm{molar}$ equivalents of NbHul5g $(n=3, \operatorname{SEM}, P<0.05)$, NbSyn2 $(n=3$, SEM, $P<0.01)$, or NbSyn87 $(n=3, \operatorname{SEM}, P<0.001)$, and incubation with the cells for $24 \mathrm{~h}$ 
containing NbSyn2 and NbSyn87 than in the presence of NbHul5g, supporting the sm-FRET findings on the rates of interconversion. Subsequently, we measured whether or not the aggregates generated during the $29 \mathrm{~h}$ incubation period in the presence of aS-specific nanobodies were more degradable in comparison to the aggregates formed in the presence of the control nanobody by exposing these samples to the $26 \mathrm{~S}$ proteasome for $24 \mathrm{~h}$, and quantifying the numbers of non-degraded oligomers by sm-FRET, as described fully in Additional file 2: Supplementary Information. The results of this experiment indicate that the oligomer populations formed in the presence of the two specific nanobodies could be degraded by the proteasome to a greater extent than the aggregates formed in the presence of the control nanobody (Fig. 4b).

We then investigated if the inhibitory effect of the nanobodies at early stages of the aggregation reaction could result in a change in oligomer-induced cellular damage. High-FRET oligomers have been found in previous studies to induce significantly higher levels of cellular toxicity in rat mid-brain neuronal cultures than the low-FRET species [13, 37, 42]. Aliquots were extracted from samples of aS undergoing aggregation in the absence or presence of nanobodies after incubation for $29 \mathrm{~h}$, and the production of reactive oxygen species (ROS) was measured following the application of these solutions to primary co-cultures of neurons and astrocytes (Additional file 2: Supplementary Information). The results show that the presence of NbSyn87 or NbSyn2 led to a significantly reduced rate of ROS production in comparison to the samples generated in their absence or in the presence of the control nanobody NbHul5g (Fig. 4c). This observed reduction in the oligomer-induced ROS indicates a reduced proportion of toxic high-FRET oligomers and supports the sm-FRET and proteinase $\mathrm{K}$ digestion results described above. We also investigated the ability of aS oligomer populations prepared in the presence or absence of nanobodies to induce cell death (see Additional file 2: Supplementary Information for details).

We found that the incubation of cells with aS aggregated in the presence of either NbSyn 87 or NbSyn2 led to a significant reduction in cell death in comparison to the incubation of aS alone (Fig. 4d), indicating in the former the presence of a lower proportion of high-FRET compared to low-FRET oligomers. Apart from the significantly reduced cytotoxicity upon application of nanobody-bound oligomers in comparison to the oligomers of aS alone in ROS and cell death assays, it was found that the samples containing NbSyn 2 reduced the cytotoxicity more effectively in comparison to NbSyn 87 (Fig. 4c, d). While the overall reduction of cytotoxicity in comparison to the aS-only samples is entirely consistent with the measured ability of NbSyn 87 and NbSyn2 to convert toxic high-FRET oligomers to low-FRET oligomers in aqueous solution, the difference between the two aS-specific nanobodies suggests the influence of other factors in the cell milieu in addition to the structure of aS oligomers. The lower protective effect of NbSyn87 compared to NbSyn2 in these experiments might be due to the high positive charge of NbSyn 87 ( $\mathrm{pI}>9.0)$ and increased interaction with the cell membranes, as well as due to its ability to abolish aS fibril formation leading to the increased oligomer load. More detailed comparison of the reduction of toxicity by NbSyn2 and NbSyn87 in the cellular environment remains to be made in future studies.

Finally, we tested whether or not the oligomers formed in the presence of the aS-specific nanobodies reduced the pro-inflammatory activation of microglial cells by quantifying the concentration of the released proinflammatory cytokine, the tumor necrosis factor alpha (TNF-a) protein [43], upon their incubation with aS solutions after aggregation for $29 \mathrm{~h}$. The experimental procedure is described in Additional file 2: Supplementary Information, and the results show that the application of aS solutions aggregated with NbSyn2 and NbSyn 87 resulted in a significantly reduced activation of microglia in comparison to aS alone and/or NbHul5g-containing samples (Fig. 4e).

Taken together, the comparative assays in Fig. 4 indicate a lower average stability of the aS oligomer populations generated in the presence of the aS-specific nanobodies and a reduced propensity to cause cellular damage. These findings are completely consistent with the conclusion that the population of the more toxic high-FRET oligomer type is reduced during the aggregation process of aS in the presence of NbSyn 87 and NbSyn2.

\section{Discussion}

In this study, we have characterized the key molecular steps that are altered by the aS-specific nanobodies, NbSyn2 and NbSyn87, during the aggregation reaction of aS. We have observed a clear effect of NbSyn2 and NbSyn87 on the formation of aS fibrils and their resulting morphology. In addition, using single-molecule fluorescence measurements we have identified a selective action of the nanobodies at the earliest stages of the aggregation reaction associated with the formation of toxic oligomeric species. In particular, both nanobodies were found to slow down the conformational conversion from less stable lowFRET oligomers to more stable high-FRET oligomers. Moreover, we observed that both NbSyn2 and NbSyn 87 are able rapidly to convert pre-formed high-FRET oligomers to low-FRET species, indicating that the high-FRET oligomers are destabilized upon the binding of these nanobodies. These effects are associated with a significant reduction of cytotoxicity induced by the oligomers. 
The ability of the nanobodies to promote the direct conformational conversion from more toxic to less toxic oligomeric species demonstrated in this study indicates the existence of an influential and unexplored mode of action of nanobodies or antibodies. Previously established modes of action of antibodies and nanobodies in vitro have included those that alter the equilibrium between fibrils, oligomers, and monomers [44], which can take a finite length of time to make sufficient changes in oligomer concentrations, and those that target oligomers to decrease their binding to cell membranes [11, 45]. The rapid conformational conversion by nanobodies of toxic oligomers to less toxic oligomers identified in our experiments has a direct effect on their ability to cause cellular damage. The results suggest, therefore, that the ability of nanobodies to promote conformational conversion of oligomers could have important potential for the development of therapeutic strategies. Since our study has been performed in vitro and at high concentrations of $\mathrm{aS}$, it will be of interest to elucidate the role of this mechanism under a more complex biological environment in vivo in future investigations.

The ability of the nanobodies to destabilize high-FRET oligomers observed here may be due to a combination of their significantly smaller size than conventional antibodies [31] and their binding to the accessible C-terminal region of the protein $[33,34]$. Antibodies binding to the $\mathrm{C}$-terminal region of aS have previously been shown to decrease the production of highly aggregation-prone truncated forms of aS lacking the C-terminal region [46]. Thus, steric effects could be an important feature in enabling the smaller nanobodies to interact more effectively with oligomers than larger antibodies and further comparison of the action of nanobodies and conventional antibodies is warranted. Optimization of this destabilization effect in future work by probing the nanobody binding site in further detail may be possible through approaches that can distinguish between different oligomer structures, and hence measure the extent of destabilization.

\section{Conclusions}

This study has involved the detailed biophysical characterization of the role of aS-specific nanobodies on the aggregation of $a S$ in vitro, including the use of single-molecule fluorescence techniques. It shows that nanobodies targeting the $\mathrm{C}$-terminal region inhibit the aggregation of aS not only by inhibiting its aggregation and elongation processes, but also by inhibiting the conformational conversion of oligomers formed prior to fibril formation. The latter effect leads to the decrease in oligomer stability and significantly reduces the cellular damage resulting from the effects of oligomeric species. Our work reveals an important mode of action of nanobodies, namely their ability rapidly to convert already formed toxic oligomers to less toxic ones. Such a mode of action has the potential to form the basis for a therapeutic strategy to combat PD and related protein misfolding conditions.

\section{Methods \\ Expression and purification of nanobodies and aS}

NbSyn87, NbSyn2, and NbHul5g were expressed in the periplasm of $E$. coli cells and purified using immobilized metal affinity chromatography and size-exclusion chromatography as previously described [33]. The amino acid sequences of NbSyn87 and NbSyn2 were as previously reported [34]. The sequence and structure of NbHul5g and the details of the construction of its more stable variant NbHul5g has been previously reported [39]. Expression and purification of wt and the alanine to cysteine mutant at position 90 (A90C) aS were carried out according to published protocols [47].

\section{ThT aggregation assays}

Solutions of $600 \mu \mathrm{L}$ of wt aS at a concentration of $70 \mu \mathrm{M}$ either with or without 2 molar equivalents of $\mathrm{NbSyn} 87$, NbSyn2, or NbHul5g in PBS buffer (10 mM phosphate, $\mathrm{pH} 7.5,100 \mathrm{mM} \mathrm{NaCl}, 2 \mathrm{mM}$ EDTA, and $0.1 \% \mathrm{NaN}_{3}$ ) were incubated for a period of $107 \mathrm{~h}$ at $37{ }^{\circ} \mathrm{C}$ with constant agitation at $200 \mathrm{rpm}$ (New Brunswick Scientific Innova 43). Aliquots of $5 \mu \mathrm{L}$ were removed at various time points and added to a $95 \mu \mathrm{L}$ ThT solution $(20 \mu \mathrm{M})$ in PBS buffer. ThT fluorescence was measured using a Cary Eclipse fluorimeter (Varian) with excitation and emission wavelengths of 440 and $480 \mathrm{~nm}$, respectively. The mean maximum intensities from triplicate measurements were reported.

\section{AFM measurements}

The end-point samples from the aggregation procedures used for the ThT assay were visualized using AFM imaging. Cleaved mica surfaces were incubated for 1 min with $10 \mu \mathrm{L}$ of $0.5 \%$ (v/v) (3-aminopropyl)triethoxysilane (Sigma-Aldrich) in deionized water (Milli-Q). Then, the surfaces were rinsed three times with $1 \mathrm{~mL}$ of deionized water (Milli-Q) and dried with a gentle stream of nitrogen. For each sample, $10 \mu \mathrm{L}$ of the solution was deposited on the positively functionalized surface. The droplet was incubated for $10 \mathrm{~min}$, then rinsed with $1 \mathrm{~mL}$ of deionized water (Milli-Q) and dried with a gentle stream of nitrogen; all procedures were carried out at room temperature. AFM maps were acquired using JPK Nanowizard 2 system operating in tapping mode and equipped with a silicon tip (Micromasch, $2 \mathrm{Nm}^{-1}$ ) with a nominal radius of $10 \mathrm{~nm}$. Image flattening was 
performed by SPIP (Image Metrology) software. Analysis of mean fibril heights was carried out as previously reported [48] and described in detail in Additional file 2: Supplementary Information.

\section{aS labeling}

The A90C mutant variant of aS was labeled with either maleimide-modified AF488 or AF594 dyes (Life Technologies) via the cysteine thiol moiety as previously reported $[13,49]$. The labeled protein was purified from the excess of free dye using a P10 desalting column with Sephadex G25 matrix (GE Healthcare) and concentrated using Amicon Ultra Centricons (Millipore), divided into aliquots, flash frozen and stored at $-80^{\circ} \mathrm{C}$. Each aliquot was thawed immediately and used only once.

\section{Estimation of the concentration of free aS}

The concentration of free $\mathrm{aS}$ was estimated as the difference between the total starting concentration of aS and the concentration of bound $\mathrm{aS}$ in the presence of nanobodies, calculated according to:

$$
\begin{aligned}
{[a s]_{\text {bound }}=\frac{1}{2}([a s]} & +[N b]+K_{d} \\
& \left.-\sqrt{\left([a s]+[N b]+K_{d}\right)^{2}-4[a s][N b]}\right)
\end{aligned}
$$

where $[a s]_{\text {bound }}$ is the concentration of bound aS, [as] and $[\mathrm{Nb}]$ are the starting concentrations of aS and nanobody, and $K_{d}$ is the corresponding dissociation constant [34].

\section{Sm-FRET experiments}

The experiments were carried out according to previously published procedures [37, 38], utilizing a custombuilt single-molecule setup as already described [50] and microfluidic devices fabricated as previously reported [38]. The single-molecule instrumentation has been previously described in detail [37]. For the aggregation reactions, equimolar concentrations of AF488- and AF594-labeled A90C aS (AF488-aS and AF594-aS) in PBS were combined to a final volume of $300 \mu \mathrm{L}$, bringing the total aS concentration to $70 \mu \mathrm{M}$, either in the presence or absence of $140 \mu \mathrm{M}$ of unlabeled NbSyn87, NbSyn2, or NbHul5g. At least three separate samples were prepared and analyzed at each protein combination. The solutions were incubated in the dark for up to $50 \mathrm{~h}$ under the same incubation conditions as the samples for ThT assays $\left(37^{\circ} \mathrm{C}\right.$, with agitation). Aliquots were withdrawn at regular time intervals and diluted $2.5 \times 10^{5}$ fold by serial dilution in either PBS buffer or deionized water (Milli-Q) to give a concentration suitable for analysis in the single molecule regime. Immediately upon dilution, the solution was passed at a constant rate of $2 \mathrm{~cm} \mathrm{~s}^{-1}$ (syringe pump PHD2000, Harvard Apparatus) through a channel of a microfluidic device mounted on the single-molecule confocal microscope (Nikon Eclipse Ti-U). The $488 \mathrm{~nm}$ laser beam was focused into the center of the channel $(10 \mu \mathrm{m}$ along vertical direction). The experimental data consisted of synchronous time-binned fluorescence output from the donor (AF488) and the acceptor (AF594) channels, acquired for $400 \mathrm{~s}$ per aliquot (80 frames, 100,000 bins frame $^{-1}, 50 \mu \mathrm{s}$ bin-width). The absence of oligomer dissociation during sm-FRET detection was previously confirmed [37], and the estimated oligomer concentrations were previously found to agree with the oligomer concentrations at bulk conditions [13].

\section{Sm-FRET data analysis}

The resulting sm-FRET data were analyzed as previously reported [37, 38], using custom-written Igor Pro code (Wavemetrics). Time-bins with intensities above 15 photons.bin ${ }^{-1}$ were selected in both the donor and acceptor channel (AND criterion) [51]. Simultaneous fluorescence bursts above the threshold were assigned to oligomeric events and the non-simultaneous donor bursts above the threshold correspond to monomeric aS. The intensities of the selected photon bursts were corrected for the crosstalk from donor to acceptor channel (13\%), and the autofluorescence in the acceptor channel $\left(1.3\right.$ photons.bin $\left.{ }^{-1}\right)$. For each oligomeric burst, the two key parameters calculated were the apparent size of the oligomer (Size) and the FRET efficiency $(E)$, defined as:

$$
\begin{aligned}
E & =\frac{I_{A}}{I_{A}+\gamma I_{D}} \\
\text { Size } & =2 \times \frac{I_{D}+\gamma^{-1} I_{A}}{I_{\text {monomer }}}
\end{aligned}
$$

where $I_{D}$ is the donor intensity in the presence of an acceptor, $I_{A}$ is the acceptor intensity, $\gamma$ is the gamma factor, which corrects for the differences in detection efficiencies of the two fluorescent probes and their quantum yields $(\gamma=0.99)$, and $I_{\text {monomer }}$ is the mean monomer brightness, averaged over all of the noncoincident bursts in the donor channel. FRET efficiency and apparent size distributions were represented as histograms with bin-widths of 0.05 and 1 , respectively. The presence of undetectable singly-colored or fluorescently quenched oligomers was assumed to have no significant effect on the data, based on our previous works [13, 38]. Large species, either those apparently greater than 150 monomers in size or occupying consecutive time-bins, 
were assumed to be due to fibrillar species and were excluded from the analysis, as reported in our previous study [38]. Subsequently, both the monomer and the oligomer bursts were converted into corresponding bulk aS concentrations by taking into account the dilution factor, as previously reported [37].

\section{Additional files}

Additional file 1: Figure S1. Additional results. (a) AFM images of starting monomeric solutions of wild-type (wt) aS prior to incubation with agitation. (b) Total internal reflection fluorescence microscopy results. Left: representative sum-image in the ThT emission channel (100 frames) and the corresponding reconstruction image in the NR channel (2000 frames). Right: comparison of the total numbers of aggregates and percent of ThT-active aggregates formed in the wt aS-only and wt aS + nanobody solutions at the same time-point of the aggregation process. (c) Scatter plots and statistical comparison of the distributions of average fibril heights derived from AFM maps (Fig. 1b, c, main text). (d) Quartz crystal microbalance recordings using as $(21 \mu \mathrm{M})$, nanobody alone $(21 \mu \mathrm{M})$ or $1: 1$ mixture of aS with nanobody $(21 \mu \mathrm{M}: 21 \mu \mathrm{M})$ or control peptide (42 $\mu \mathrm{M})$. (TIF $4648 \mathrm{~kb}$ )

Additional file 2: Supplementary Information. (PDF $462 \mathrm{~kb}$ ) Additional file 3: Figure S2. Results of control bulk ThT experiments and TEM imaging of labeled aS. (a) Progression of fibril formation, monitored by ThT fluorescence emission from either unlabeled wild-type (black) or AF-594 labeled aS at $70 \mu \mathrm{M}(n=3$, SD). (b) TEM images of aggregates formed in $70 \mu \mathrm{M}$ 1:1 AF488:AF594 dual-labeled aS solutions. Top: labeled as solutions at different time-points during aggregation. Bottom: as solutions in the presence of $140 \mu \mathrm{M}$ of unlabeled NbHul5g, NbSyn2, and NbSyn87 after more than 100 h incubation with agitation. Large amyloid fibrils and fibrillar fragments were observed in all samples at this time, except in the presence of NbSyn87, where short protofibrils were present along with oligomeric aggregates. (TIF 4331 kb)

Additional file 4: Figure S3. Kinetic analysis of aS aggregation. (a) Schematic representation of the nucleation-conversion-polymerization model. Monomeric as form low-FRET oligomers with rate constant $k_{n}$ and an average reaction order $n_{c}$. Low-FRET oligomers convert into highFRET oligomers by a first-order reaction with a rate constant $k_{1}{ }^{c}$, which is followed by a first-order conversion to fibrils, with a rate constant $k_{2}{ }^{c}$. Once formed, fibrils grow by monomer addition with a lengthindependent rate constant $k_{e}$. The conversion steps between oligomer types are treated as size independent, and $k_{1}{ }^{c}$ is set to be equal to $k_{2}{ }^{c}$. First-order reverse conversion reactions from high-FRET to low-FRET oligomers and from low-FRET oligomers to monomers are introduced, with rate constants $k_{1}^{c}$ and $k_{n}$, respectively. (b-e) This model was fitted globally to the kinetic data of aS aggregation at $70 \mu \mathrm{M}$ in the absence or the presence of $140 \mu \mathrm{M}$ of nanobodies. The global fits (dashed lines) were performed with parameters $k_{n}=(1.0 \pm 0.5) \times 10^{-3} \mathrm{~h}^{-1}, k_{e}=0.16$ $\pm 0.08 \mu \mathrm{M}^{-1} \mathrm{~h}^{-1}, \mathrm{k}_{1}{ }^{c}=k_{2}{ }^{c}=0.12 \pm 0.04 \mathrm{~h}^{-1}, \widetilde{k_{n}}=\widetilde{k_{1}^{c}}=0 \mathrm{~h}^{-1}, n_{c}=1 \pm$ $0.1, m_{\text {tot }}=70 \mu \mathrm{M}$ for reactions containing as only and as with $\mathrm{NbHul} 5 \mathrm{~g}$. The reverse reaction from high-FRET oligomers to low-FRET oligomers was found to be accelerated in the presence of NbSyn2 and NbSyn87, with $\tilde{k_{n}}=0.2 \pm 0.1 \mathrm{~h}^{-1}$ and $\widetilde{k_{1}^{c}}=1 \pm 0.5 \mathrm{~h}^{-1}$ for aS aggregation in the presence of NbSyn2, and with $\tilde{k_{n}}=0.25 \pm 0.1 \mathrm{~h}^{-1}$ and $\widetilde{k_{1}^{c}}=10 \pm 5 \mathrm{~h}^{-1}$ for aS aggregation in the presence of NbSyn87, with all remaining parameters unchanged. (f) Predictions of the concentrations of low-FRET and high-FRET oligomers during $100 \mathrm{~h}$ of as aggregation in the presence or absence of nanobodies. (TIF $710 \mathrm{~kb}$ )

Additional file 5: Figure S4. Representative FRET efficiency histograms from the 'reverse' sm-FRET experiments, analogous to those shown in Fig. 3 (main text). (a) Pre-formed high-FRET oligomers were formed in a forward incubation of monomeric aS (70 $\mu \mathrm{M}$ in PBS, $29 \mathrm{~h}$, shaking at $37^{\circ} \mathrm{C}$ ). To the pre-formed oligomer solutions, either $1,0.5$, or 0.25 molar equivalents of nanobodies, NbSyn87 (b) or NbSyn2 (c), were added and sm-FRET detection was carried out within 5 min after the addition. In the case of 0.25 equivalents, the samples were further incubated at $37{ }^{\circ} \mathrm{C}$ under quiescent conditions (in low-binding test-tubes), and sm-FRET analysis repeated. (TIF 877 kb)

Additional file 6: Figure S5. Representative contour plots of FRET efficiency and size after proteinase $K$ digestion of 29-h time-points by different concentrations of proteinase K (Fig. 4a, main text). (a) Control sample containing NbHul5g is less degradable in comparison to the samples prepared in the presence of NbSyn2 (b) and NbSyn87 (c), as indicated by the presence of higher oligomer fraction remaining in the sample upon incubation with proteinase $\mathrm{K}$. This is consistent with the presence of high-FRET oligomers in the sample. (TIF $1028 \mathrm{~kb}$ )

Additional file 7: Supporting Data Values. (XLSX $27 \mathrm{~kb}$ )

Additional file 8: Figure S6. Representative result from the reactive oxygen species measurements presented in main text, Fig. 4c. Application of $500 \mathrm{nM}$ of AF-labeled aS solution induced an increase in the ratio of dihydroethydium (HEt) fluorescence between its oxidized and non-oxidized forms. The time when aS was applied is marked with the grey bracket on the plot. A higher increase in HEt ratio is observed upon application of aS solutions containing control $\mathrm{NbHul} 5 \mathrm{~g}$, suggesting that oligomers formed in its presence are more damaging in comparison to the oligomers formed in the presence of aS-specific nanobodies. (TIF $108 \mathrm{~kb}$ )

\section{Acknowledgements}

We are grateful to Ewa Klimont and Swapan Preet for the expression and purification of $\mathrm{A} 90 \mathrm{C}$ aS, and thank Dr. Yu Ye for the reagents used in the proteasome degradation assays.

\section{Funding}

MI was funded by a Dr Tayyeb Hussain Scholarship and the ERC (669237). LH was funded by a China Scholarship Council and NSFC 11204150. TG was a recipient of a studentship from Parkinson's UK (H-0903). EDG was supported by the Medical Research Council (MRC G1002272). DK was funded by the ERC (669237) and the Royal Society, CMD, TPJK and MV by the Wellcome Trust.

\section{Availability of data and materials}

All experimental methods and results generated during this study are included in the article and in Additional file 2: Supplementary Information (SI.pdf), available online. The individual data values for the datasets in Figs. 1 and 4 are included as an Additional file 7: Supporting Data Values.xls.

\section{Authors' contributions}

MI designed and performed the single-molecule fluorescence experiments and data analysis. EDG performed the nanobody preparation, bulk thioflavin T assays, and aided with single-molecule experiments. LH performed the kinetic analysis. $\mathrm{MHH}$ aided with the single-molecule experiments and the design of the project. MHL, MLC, and CDH performed the cell experiments. SG and CEB designed the cell experiments. FSR performed the AFM measurements and analysis. AKB performed the quartz crystal microbalance experiments. TG performed the nanobody preparation. JEL and SFL performed total internal reflection fluorescence microscopy imaging and data analysis. TPJK and MV supervised the study. CMD, EDG, and DK designed and supervised the study. MI, EDG, DK, and CMD wrote the paper, with contributions from all authors. All authors read and approved the final manuscript.

\section{Competing interests}

The authors declare no competing financial interests.

\section{Consent for publication}

Not applicable.

Ethics approval and consent to participate Not applicable.

\section{Publisher's Note}

Springer Nature remains neutral with regard to jurisdictional claims in published maps and institutional affiliations. 


\section{Author details}

'Department of Chemistry, University of Cambridge, Lensfield Road, Cambridge CB2 1EW, UK. ${ }^{2}$ Zhou Pei-Yuan Center for Applied Mathematics, Tsinghua University, Beijing 100084, China. ${ }^{3}$ Wellcome Trust-Medical Research Council Cambridge Stem Cell Institute, University of Cambridge, Cambridge CB2 1QR, UK. ${ }^{4}$ Department of Molecular Neuroscience, University College London, Institute of Neurology, Queen Square, London WC1N 3BG, UK. ${ }^{5}$ Department of Veterinary Medicine, University of Cambridge, Madingley Road, Cambridge CB3 OES, UK. 'Present address: Healx Ltd., St John's Innovation Centre, Cowley Road, Cambridge CB4 OWS, UK. ${ }^{7}$ Present address: Astra Zeneca, Innovative Medicines Discovery Sciences Unit 310, Darwin Building, Cambridge Science Park, Milton Road, Cambridge CB4 OWG, UK. ${ }^{8}$ Present address: Institute of Physical Biology, University of Düsseldorf, Universitätsstr. 1, 40225 Düsseldorf, Germany.

Received: 11 April 2017 Accepted: 6 June 2017

Published online: 03 July 2017

\section{References}

1. Baba M, Nakajo S, Tu PH, Tomita T, Nakaya K, Lee VM, Trojanowski JQ, Iwatsubo T. Aggregation of alpha-synuclein in Lewy bodies of sporadic Parkinson's disease and dementia with Lewy bodies. Am J Pathol. 1998;152: 879-84.

2. Newell KL, Boyer P, Gomez-Tortosa E, Hobbs W, Hedley-Whyte ET, Vonsattel JP, Hyman BT. Alpha-synuclein immunoreactivity is present in axonal swellings in neuroaxonal dystrophy and acute traumatic brain injury. J Neuropathol Exp Neurol. 1999;58:1263-8.

3. Wakabayashi K, Yoshimoto M, Tsuji S, Takahashi H. Alpha-synuclein immunoreactivity in glial cytoplasmic inclusions in multiple system atrophy. Neurosci Lett. 1998;249:180-2.

4. Arawaka S, Saito Y, Murayama S, Mori H. Lewy body in neurodegeneration with brain iron accumulation type 1 is immunoreactive for alpha-synuclein. Neurology. 1998;51:887-9.

5. Spillantini M, Crowther R, Jakes R, Cairns N, Lantos P, Goedert M. Filamentous alpha-synuclein inclusions link multiple system atrophy with Parkinson's disease and dementia with Lewy bodies. Neurosci Lett. 1998; 251:205-8.

6. Del Tredici K, Braak H. Review: Sporadic Parkinson's disease: development and distribution of a-synuclein pathology. Neuropathol Appl Neurobiol. 2016:42:33-50

7. Goedert M, Masuda-Suzukake M, Falcon B. Like prions: the propagation of aggregated tau and a-synuclein in neurodegeneration. Brain. 2017;140(Pt 2): 266-78.

8. Fink AL. The aggregation and fibrillation of alpha-synuclein. Acc Chem Res. 2006:39:628-34.

9. Buell AK, Galvagnion C, Gaspar R, Sparr E, Vendruscolo M, Knowles TP, Linse $\mathrm{S}$, Dobson CM. Solution conditions determine the relative importance of nucleation and growth processes in a-synuclein aggregation. Proc Natl Acad Sci U S A. 2014;111:7671-6.

10. Conway K, Lee S, Rochet J, Ding T, Williamson R, Lansbury P. Acceleration of oligomerization, not fibrillization, is a shared property of both alphasynuclein mutations linked to early-onset Parkinson's disease: Implications for pathogenesis and therapy. Proc Natl Acad Sci U S A. 2000;97:571-6.

11. Kalia LV, Kalia SK, McLean PJ, Lozano AM, Lang AE. a-Synuclein oligomers and clinical implications for Parkinson disease. Ann Neurol. 2013;73:155-69.

12. Volles MJ, Lee SJ, Rochet JC, Shtilerman MD, Ding TT, Kessler JC, Lansbury PT. Vesicle permeabilization by protofibrillar alpha-synuclein: implications for the pathogenesis and treatment of Parkinson's disease. Biochemistry. 2001; 40:7812-9.

13. Cremades N, Cohen SI, Deas E, Abramov AY, Chen AY, Orte A, Sandal M, Clarke RW, Dunne P, Aprile FA, et al. Direct observation of the interconversion of normal and toxic forms of a-synuclein. Cell. 2012;149:1048-59.

14. Roberts RF, Wade-Martins R, Alegre-Abarrategui J. Direct visualization of alpha-synuclein oligomers reveals previously undetected pathology in Parkinson's disease brain. Brain. 2015;138:1642-57.

15. Näsström T, Fagerqvist $T$, Barbu M, Karlsson M, Nikolajeff F, Kasrayan A, Ekberg M, Lannfelt L, Ingelsson M, Bergström J. The lipid peroxidation products 4-oxo-2-nonenal and 4-hydroxy-2-nonenal promote the formation of a-synuclein oligomers with distinct biochemical, morphological, and functional properties. Free Radic Biol Med. 2011;50:428-37.
16. Diógenes MJ, Dias RB, Rombo DM, Vicente Miranda H, Maiolino F, Guerreiro P, Näsström T, Franquelim HG, Oliveira LM, Castanho MA, et al. Extracellular alpha-synuclein oligomers modulate synaptic transmission and impair LTP via NMDA-receptor activation. J Neurosci. 2012;32:11750-62.

17. Bellucci A, Navarria L, Zaltieri M, Missale C, Spano P. a-Synuclein synaptic pathology and its implications in the development of novel therapeutic approaches to cure Parkinson's disease. Brain Res. 2012;1432:95-113.

18. George S, Brundin P. Immunotherapy in Parkinson's disease: micromanaging alpha-synuclein aggregation. J Parkinsons Dis. 2015;5:413-24.

19. Valera E, Spencer B, Masliah E. Immunotherapeutic approaches targeting amyloid- $\beta$, $\alpha$-synuclein, and tau for the treatment of neurodegenerative disorders. Neurotherapeutics. 2016;13:179-89.

20. Bergström AL, Kallunki P, Fog K. Development of passive immunotherapies for synucleinopathies. Mov Disord. 2016;31:203-13.

21. De Genst E, Messer A, Dobson CM. Antibodies and protein misfolding: from structural research tools to therapeutic strategies. Biochim Biophys Acta. 1844;2014:1907-19.

22. Emadi S, Liu R, Yuan B, Schulz P, McAllister C, Lyubchenko Y, Messer A, Sierks MR. Inhibiting aggregation of alpha-synuclein with human single chain antibody fragments. Biochemistry. 2004;43:2871-8.

23. Emadi S, Kasturirangan S, Wang MS, Schulz P, Sierks MR. Detecting morphologically distinct oligomeric forms of alpha-synuclein. J Biol Chem. 2009;284:11048-58.

24. Emadi S, Barkhordarian H, Wang MS, Schulz P, Sierks MR. Isolation of a human single chain antibody fragment against oligomeric alpha-synuclein that inhibits aggregation and prevents alpha-synuclein-induced toxicity. J Mol Biol. 2007;368:1132-44.

25. Zhou C, Emadi S, Sierks MR, Messer A. A human single-chain Fv intrabody blocks aberrant cellular effects of overexpressed alpha-synuclein. Mol Ther 2004;10:1023-31.

26. Lynch SM, Zhou C, Messer A. An scFv intrabody against the nonamyloid component of alpha-synuclein reduces intracellular aggregation and toxicity. J Mol Biol. 2008;377:136-47.

27. Näsström T, Gonçalves S, Sahlin C, Nordström E, Screpanti Sundquist V, Lannfelt L, Bergström J, Outeiro TF, Ingelsson M. Antibodies against alphasynuclein reduce oligomerization in living cells. PLoS One. 2011;6:e27230.

28. Bisaglia M, Mammi S, Bubacco L. Structural insights on physiological functions and pathological effects of alpha-synuclein. FASEB J. 2009;23:329-40.

29. Der-Sarkissian A, Jao C, Chen J, Langen R. Structural organization of alpha-synuclein fibrils studied by site-directed spin labeling. J Biol Chem. 2003;278:37530-5.

30. Murray IV, Giasson BI, Quinn SM, Koppaka V, Axelsen PH, Ischiropoulos H, Trojanowski JQ, Lee VM. Role of alpha-synuclein carboxy-terminus on fibril formation in vitro. Biochemistry. 2003:42:8530-40.

31. Hamers-Casterman C, Atarhouch T, Muyldermans S, Robinson G, Hamers C, Songa EB, Bendahman N, Hamers R. Naturally occurring antibodies devoid of light chains. Nature. 1993;363:446-8.

32. Muyldermans S. Single domain camel antibodies: current status. J Biotechnol. 2001;74:277-302.

33. De Genst EJ, Guilliams T, Wellens J, O'Day EM, Waudby CA, Meehan S, Dumoulin M, Hsu ST, Cremades N, Verschueren $\mathrm{KH}$, et al. Structure and properties of a complex of a-synuclein and a single-domain camelid antibody. J Mol Biol. 2010;402:326-43.

34. Guilliams T, El-Turk F, Buell AK, O'Day EM, Aprile FA, Esbjörner EK, Vendruscolo M, Cremades N, Pardon E, Wyns L, et al. Nanobodies raised against monomeric a-synuclein distinguish between fibrils at different maturation stages. J Mol Biol. 2013;425:2397-411.

35. Butler DC, Joshi SN, Genst E, Baghel AS, Dobson CM, Messer A. Bifunctional anti-non-amyloid component a-Synuclein nanobodies are protective in situ. PLoS One. 2016;11:e0165964.

36. Mysling $\mathrm{S}$, Betzer $\mathrm{C}$, Jensen PH, Jorgensen TJ. Characterizing the dynamics of a-synuclein oligomers using hydrogen/deuterium exchange monitored by mass spectrometry. Biochemistry. 2013;52:9097-103.

37. Iljina M, Garcia GA, Horrocks MH, Tosatto L, Choi ML, Ganzinger KA, Abramov AY, Gandhi S, Wood NW, Cremades N, et al. Kinetic model of the aggregation of alpha-synuclein provides insights into prion-like spreading. Proc Natl Acad Sci U S A. 2016;113(9):E1206-15.

38. Horrocks MH, Tosatto L, Dear AJ, Garcia GA, Iljina M, Cremades N, Dalla Serra M, Knowles TP, Dobson CM, Klenerman D. Fast flow microfluidics and single-molecule fluorescence for the rapid characterization of a-synuclein oligomers. Anal Chem. 2015;87:8818-26. 
39. De Genst E, Chan PH, Pardon E, Hsu ST, Kumita JR, Christodoulou J, Menzer L, Chirgadze DY, Robinson CV, Muyldermans S, et al. A nanobody binding to non-amyloidogenic regions of the protein human lysozyme enhances partial unfolding but inhibits amyloid fibril formation. J Phys Chem B. 2013; 117:13245-58

40. Knowles TP, Shu W, Devlin GL, Meehan S, Auer S, Dobson CM, Welland ME. Kinetics and thermodynamics of amyloid formation from direct measurements of fluctuations in fibril mass. Proc Natl Acad Sci U S A. 2007; 104:10016-21.

41. Buell AK, Dobson CM, Welland ME. Measuring the kinetics of amyloid fibril elongation using quartz crystal microbalances. Methods Mol Biol. 2012;849. 101-19.

42. Deas E, Cremades N, Angelova PR, Ludtmann MH, Yao Z, Chen S, Horrocks MH, Banushi B, Little D, Devine MJ, et al. Alpha-synuclein oligomers interact with metal ions to induce oxidative stress and neuronal death in Parkinson's disease. Antioxid Redox Signal. 2016;24:376-91.

43. Stefanova N, Schanda K, Klimaschewski L, Poewe W, Wenning GK, Reindl M. Tumor necrosis factor-alpha-induced cell death in U373 cells overexpressing alpha-synuclein. J Neurosci Res. 2003;73:334-40.

44. Pain C, Dumont J, Dumoulin M. Camelid single-domain antibody fragments: uses and prospects to investigate protein misfolding and aggregation, and to treat diseases associated with these phenomena. Biochimie. 2015;111:82-106.

45. Sahin C, Lorenzen N, Lemminger L, Christiansen G, Møller IM, Vesterager LB, Pedersen L, Fog K, Kallunki P, Otzen DE. Antibodies against the C-terminus of a-synuclein modulate its fibrillation. Biophys Chem. 2017;220:34-41.

46. Games D, Valera E, Spencer B, Rockenstein E, Mante M, Adame A, Patrick C, Ubhi K, Nuber S, Sacayon P, et al. Reducing C-terminal-truncated alpha-synuclein by immunotherapy attenuates neurodegeneration and propagation in Parkinson's disease-like models. J Neurosci. 2014;34:9441-54.

47. Hoyer W, Antony T, Cherny D, Heim G, Jovin T, Subramaniam V. Dependence of alpha-synuclein aggregate morphology on solution conditions. J Mol Biol. 2002;322:383-93.

48. Ruggeri FS, Vieweg S, Cendrowska U, Longo G, Chiki A, Lashuel HA, Dietler G. Nanoscale studies link amyloid maturity with polyglutamine diseases onset. Sci Rep. 2016;6:31155.

49. Thirunavukkuarasu S, Jares-Erijman E, Jovin T. Multiparametric fluorescence detection of early stages in the amyloid protein aggregation of pyrene-labeled alpha-synuclein. J Mol Biol. 2008;378:1064-73.

50. Orte A, Clarke R, Balasubramanian S, Klenerman D. Determination of the fraction and stoichiometry of femtomolar levels of biomolecular complexes in an excess of monomer using single-molecule, two-color coincidence detection. Anal Chem. 2006;78:7707-15.

51. Ying L, Wallace M, Balasubramanian S, Klenerman D. Ratiometric analysis of single-molecule fluorescence resonance energy transfer using logical combinations of threshold criteria: A study of 12-mer DNA. J Phys Chem B. 2000;104:5171-8.

\section{Submit your next manuscript to BioMed Central and we will help you at every step:}

- We accept pre-submission inquiries

- Our selector tool helps you to find the most relevant journal

- We provide round the clock customer support

- Convenient online submission

- Thorough peer review

- Inclusion in PubMed and all major indexing services

- Maximum visibility for your research

Submit your manuscript at www.biomedcentral.com/submit

Biomed Central 